\title{
CT EVALUATION OF VARIOUS LINEAR INDICES IN CHILDREN WITH CLINICALLY SUSPECTED HYDROCEPHALUS
}

\author{
Goyal Swati1 , Goyal Sanjay², Yadav Pankaj ${ }^{3}$, Mishra Saumya ${ }^{4}$
}

${ }^{1}$ Assistant Professor, Department of Radiodiagnosis, GRMC, Gwalior.

2Member, Executive Committee, GRMC and JAH, GRMC, Gwalior.

${ }^{3}$ Associate Professor, Department of Radiodiagnosis, GRMC, Gwalior.

${ }^{4}$ Resident, Department of Radiodiagnosis, GRMC, Gwalior.

\section{ABSTRACT}

\section{BACKGROUND}

Objective- To prospectively measure and evaluate various CT linear indices namely Cella Media index (CMI), Frontal Horn Index (FHI), Evan's Index (EI) and Ventricular/Biparietal (V/BP) Ratio in children with clinically suspected hydrocephalus caused by various aetiologies.

\section{MATERIALS AND METHODS}

Axial CT scans of 53 symptomatic children were studied prospectively with proper consent. Out of these 53 patients, 31 were males and 22 were females. All patients had 2-phase examination (pre- and post-contrast studies) of the brain using the department's Siemens 128 slice somatom definition as multi-detector spiral CT. Predicated on the measurements various linear indices, notably Cella Media Index, Frontal Horn Index, Evan's index and V/BP Ratio were calculated.

\section{RESULTS}

In our study, meningitis was found to be the most common cause of hydrocephalus in children followed by HIE, aqueductal stenosis and intracranial tumours. Of these 53 cases, 32 had communicating and 21 had non-communicating hydrocephalus. We can grade hydrocephalus based on measured linear indices as mild, moderate and severe.

\section{CONCLUSION}

Our study proved that combination of various indices measured together, rather than single index is better in grading hydrocephalus. CT is a valuable tool in differentiating communicating from non-communicating hydrocephalus depending on the level of obstruction. CT is also useful in determining the aetiology of hydrocephalus in children.

\section{KEYWORDS}

Hydrocephalus, FHI, CMI, EI, V/BP Ratio.

HOW TO CITE THIS ARTICLE: Swati G, Sanjay G, Pankaj Y, et al. CT evaluation of various linear indices in children with clinically suspected hydrocephalus. J. Evolution Med. Dent. Sci. 2017;6(38):3078-3082, DOI: 10.14260/Jemds/2017/664

\section{BACKGROUND}

Suspected hydrocephalus is the most frequent referral indication in the paediatric age group for CT head. Often the discernment of ventricular enlargement, when viewing images from CT/MRI is inferred by the subjective assessment based upon reviewer's experience.[1] Though MRI has evolved as the new technique, yet visualisation of brain ventricles by CT remains the most widely accessible and affordable tool in Indian scenario.[2] CT is useful for evaluation of brain morphology including size and shape of ventricular system. ${ }^{\text {[3] }}$ Brain ventricles can be studied by taking linear, planimetric or volumetric measurements, out of which linear ratios are probably the most conveniently made and the consistent ones. ${ }^{[4]}$ Causes and imaging patterns differ depending on the age of the patient.(5) Hydrocephalus is classified into communicating and non-communicating relying on the site and aetiology of occlusion.

Financial or Other, Competing Interest: None.

Submission 16-11-2016, Peer Review 06-04-2017,

Acceptance 12-04-2017, Published 11-05-2017.

Corresponding Author:

Dr. Goyal Swati,

C/o. Dr. Sanjay Goyal, 11,

Collector's Bungalow, Gandhi Road,

Gwalior-474001, Madhya Pradesh.

E-mail: swati_97in@yahoo.com

DOI: $10.14260 /$ jemds/2017/664
Various established indices should be used to assess degree of ventricular enlargement rather than personal experience. Our aim was to assess various CT indices in children with suspected hydrocephalus.

\section{MATERIALS AND METHODS}

Axial CT scans of symptomatic children were studied prospectively with proper consent. This is a noninterventional study, appropriately approved by the ethical committee. Total of 53 patients reviewed were aged up to 10 years, referred to the Department for CT scan due to headache, somnolence, concentration difficulties or developmental delay. No other children were subjected to ionising radiation due to ethical considerations.[1] Out of these 53 patients, 31 were males and 22 were females. All patients had 2-phase examination (pre- and post-contrast studies) of the brain using the department's Siemens 128 slice Somatom definition AS multi-detector spiral CT. Based on the measurements following linear indices were calculated.

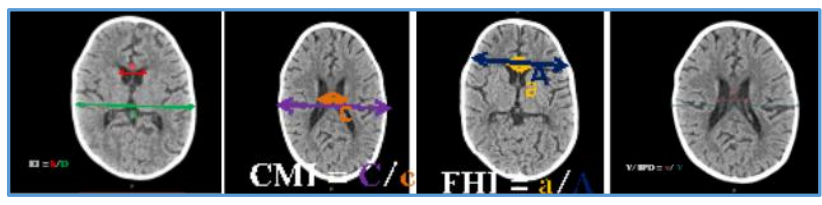

Figure 1. 1, 1.2, 1.3 and 1.4: Demonstrates Method to Measure various Linear Indices 
Cella Media Index (CMI) compares the maximum transverse diameter of skull to the maximum width of cella media. It measures $>4$ in normal cases. ${ }^{[6,7]}$

Frontal Horn Index (FHI) is a ratio that compares width of frontal horns and internal diameter of skull at the same level. It measures $<0.3$ in normal cases.[8,9,10]

Evan's index estimates the width of greatest span of frontal horns divided by the maximum width of internal diameter of the skull. Usually, it measures less than or equal to $0.29 .[3,9,10,11]$

Ventricular/Biparietal Ratio (V/BP Ratio) is computed at the mid-portion of bodies of lateral ventricles showing the maximum ventricular dilatation in axial section. It measures less than 0.26 in normal cases.[4]

\section{Exclusion Criteria}

1. History of severe head trauma.

2. Children with intraventricular and subarachnoid haemorrhage.

3. Uncooperative children.

\section{Data Analysis and Interpretation}

53 children were analysed, their age and sex distribution was studied. According to Edwards et al[12] incidence of hydrocephalus is equal in males and females, but there were more males in our study with a male: female ratio of 1.41: 1 . Of these 53 cases, 32 had communicating and 21 had noncommunicating hydrocephalus. Among these 53 patients 11 suffered from HIE, 7 each had idiopathic aqueductal stenosis and intracranial tumours, 2 each with Dandy-Walker spectrum, infarcts and TORCH infections. There were 3 more cases, each afflicted from partial agenesis of corpus callosum, granuloma and associated myelomeningocele respectively. Maximum number of 19 meningitis cases were also the part of our dissertation.

Hydrocephalus is graded according to V/BP Ratio by Chaudhary et al as $<0.26$ (Normal), $0.26-0.4$ (Mild), $0.4-0.6$ (Moderate), $0.6-0.8$ (Severe) and $>0.8$ as extreme hydrocephalus. ${ }^{[4]}$ In our research, we had 10 cases with V/BP ratio $<0.26$. In all these 10 cases, CMI was $>4$ except for two cases in which one had CMI 3.9 (minimal difference) and other had 2.3. The child with $2.3 \mathrm{CMI}$ was 2 months old and suffered from HIE, but his FHI was normal (0.3). Out of these 10 patients with normal V/BP Ratio, 3 cases had abnormal FHI of $>0.3$. Of these 10 patients, EI was in the range of 0.2 0.45 with an arithmetic mean of 0.316 . We had maximum number of 27 patients with V/BP Ratio ranging between 0.26 - 0.4, considered as mild hydrocephalus with an arithmetic mean of 0.34 . In these patients, mean CMI was 2.7 with a range of $3.5-2.3$. Likewise, mean FHI was 0.453 with a range of 0.3 - 0.6. Similarly, mean EI was 0.383 with a range of 0.23 - 0.59. We had 11 cases with V/BP Ratio between 0.4 - 0.6 with an arithmetic mean of 0.510 . In these patients, CMI ranged between $2.4-1.6$ with an average of 1.993 . Similarly, FHI in these patients had a mean of 0.510 ranging between $0.4-0.8$. The mean EI in these cases was 0.41 with a range of 0.24 - 0.7. There were only 5 cases with V/BP Ratio exceeding 0.6 with an average of 0.76 . In these cases, average CMI was 1.42 with the range of $1.9-1.2$. Likewise, FHI in these cases ranged from $0.7-1.1$ with an average of 0.88 . EI in these children ranged between $0.56-0.8$ with an arithmetic mean of 0.732 . Of these 5 cases, 4 had V/BP ratio between $0.6-0.8$ and only one child with associated myelomeningocele had the ratio of 0.9 suggestive of extreme hydrocephalus. All these 5 cases with severe hydrocephalus had non-communicating hydrocephalus and all were under 1 year of age.

V/BP Ratio $<0.26$

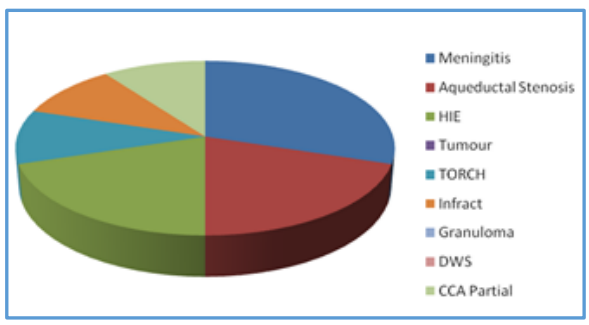

V/BP Ratio 0.26 - 0.4

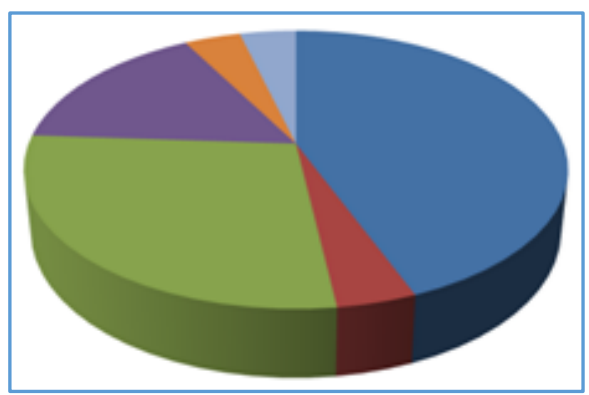

V/BP Ratio 0.4 - 0.6

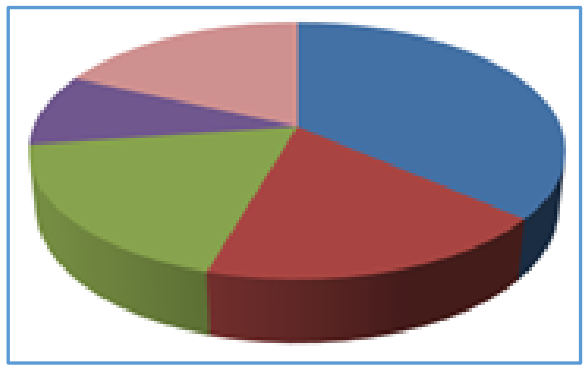

V/BP Ratio 0.6 - 0.9

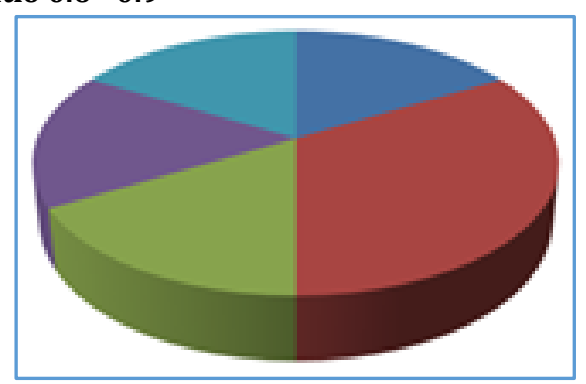

Figure 2. With Pie Chart 2.1, 2.2, 2.3 and 2.4: Illustrates Grading of Hydrocephalus based on V/BP Ratio, demonstrating various Aetiologies

In 10 patients with V/BP Ratio < 0.26, 3 had meningitis, 2 each with aqueductal stenosis and HIE respectively and 1 each afflicted from subacute infarct, TORCH and partial agenesis of corpus callosum respectively. In 27 patients with V/BP Ratio between $0.26-0.4$, maximum of 12 cases were due to meningitis, 7 had HIE, 5 with tumours and 1 each of aqueductal stenosis, granuloma and infarct respectively. In 11 patients with V/BP Ratio 0.4 - 0.6, 4 presented with meningitis, 1 had tumour and 2 each of aqueductal stenosis, 
HIE and Dandy-Walker Spectrum. In group with V/BP Ratio > 0.6 , there were 5 patients, 2 had aqueductal stenosis, 1 each with tumour and TORCH infection respectively; 1 case with V/BP Ratio approx. 0.9 had associated myelomeningocele.
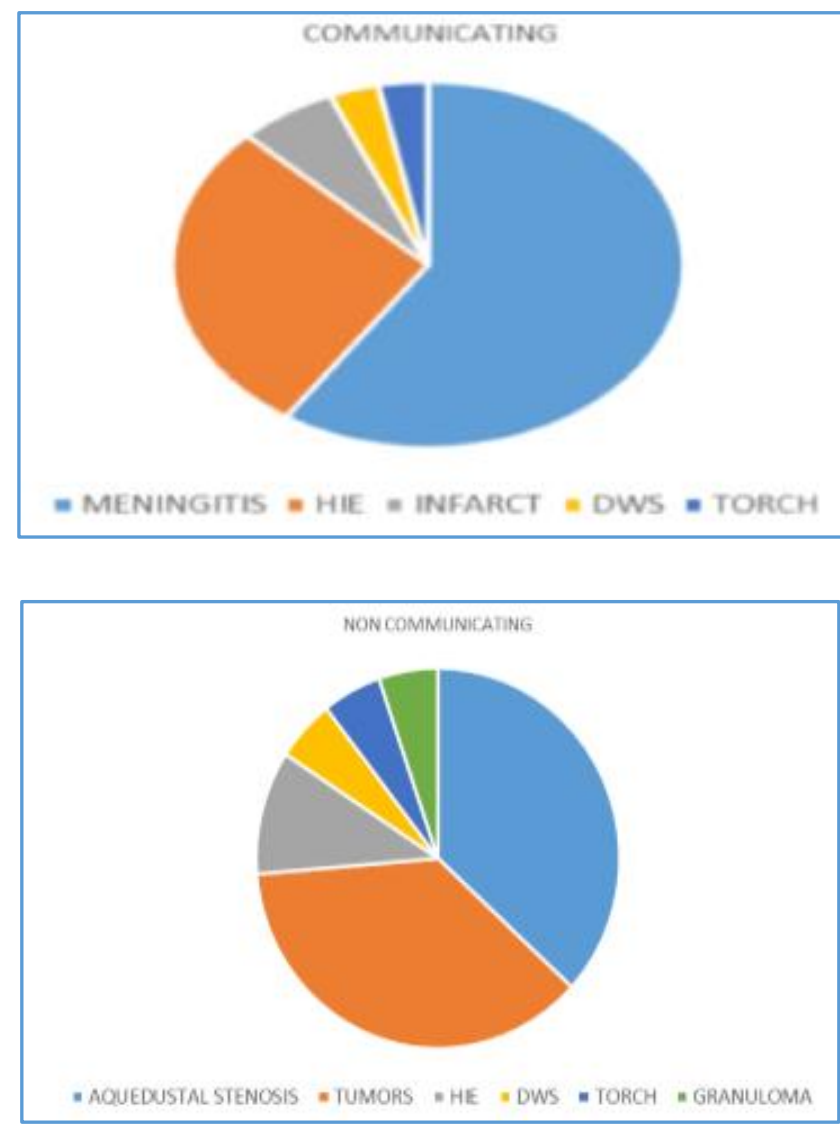

Figure 3. With Pie Charts 3.1 \& 3.2: Demonstrates Differentiation of Communicating vs Non-Communicating Hydrocephalus based on different Aetiologies

In 32 patients with communicating hydrocephalus maximum of 19 patients had meningitis, 9 cases had HIE, 2 patients had infarcts and 1 each of DWS and TORCH.

In 21 cases with non-communicating hydrocephalus maximum of equal number of 7 patients had aqueductal stenosis and tumours respectively; 2 cases had HIE and 1 each of DWS, TORCH, Granuloma, associated myelomeningocele and partial agenesis of corpus callosum respectively.

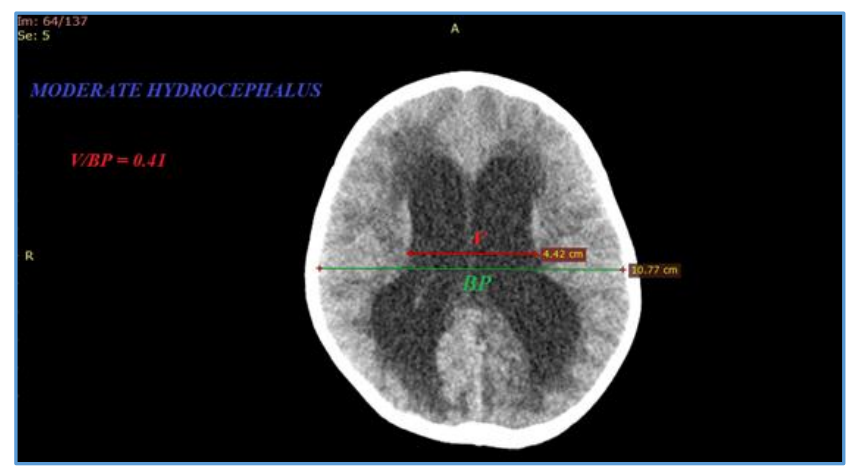

Figure 4. Illustrating Moderate Hydrocephalus with $V / B P>0.4$

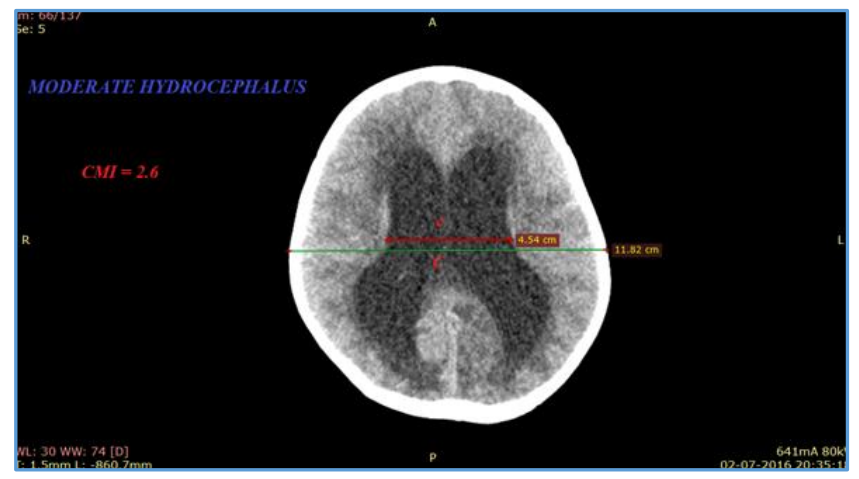

Figure 5. Illustrates Moderate Hydrocephalus with CMI $<2.4$

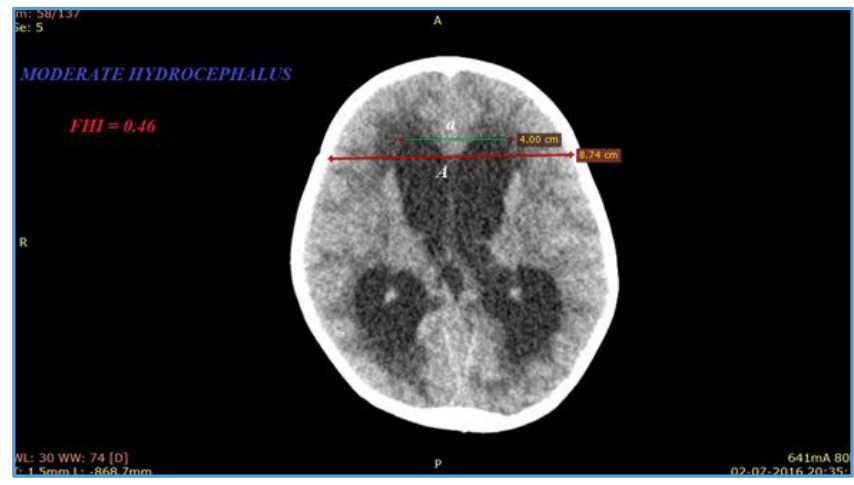

Figure 6. Illustrates Moderate Hydrocephalus with FHI > 0.4

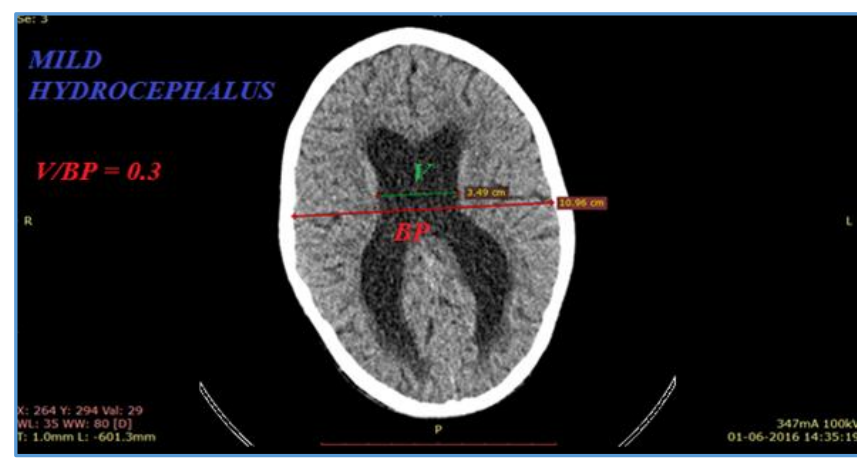

Figure 7. Illustrates Mild Hydrocephalus with $V / B P<0.4$

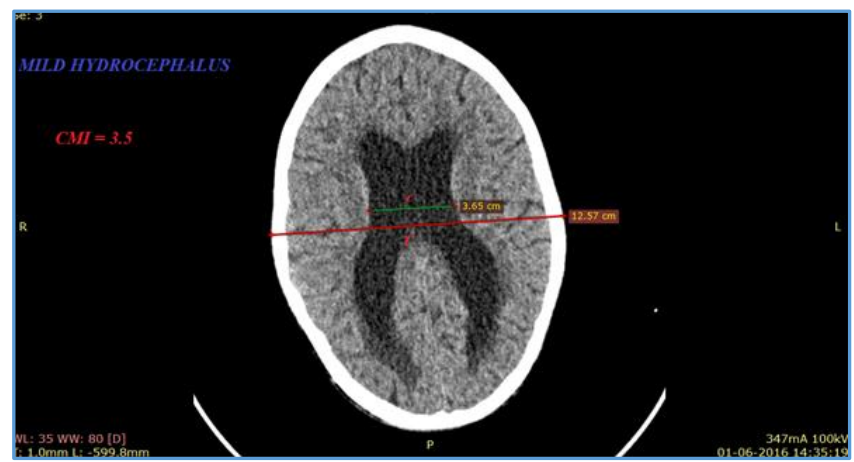

Figure 8. Illustrates mild

Hydrocephalus with CMI $>2.4$ 


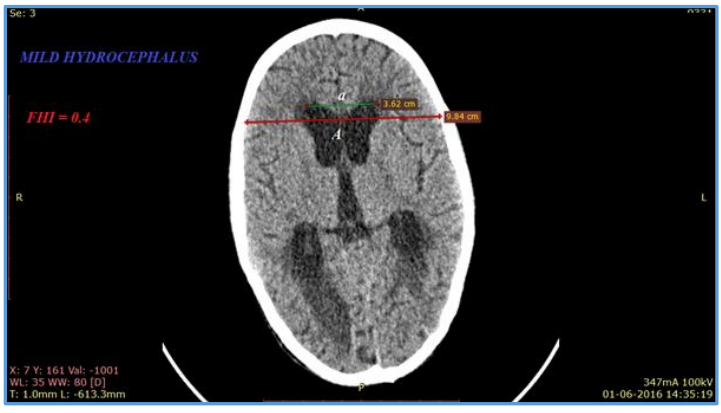

Figure 9. Illustrates mild Hydrocephalus with 0.4 FHI

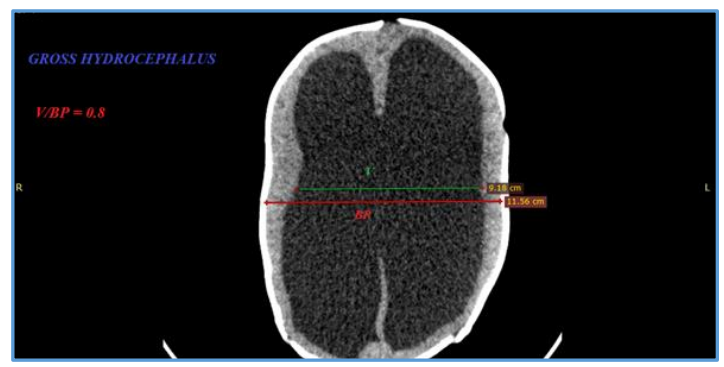

Figure 10. Illustrates Severe Hydrocephalus with $\mathrm{V} / \mathrm{BP}>0.6$

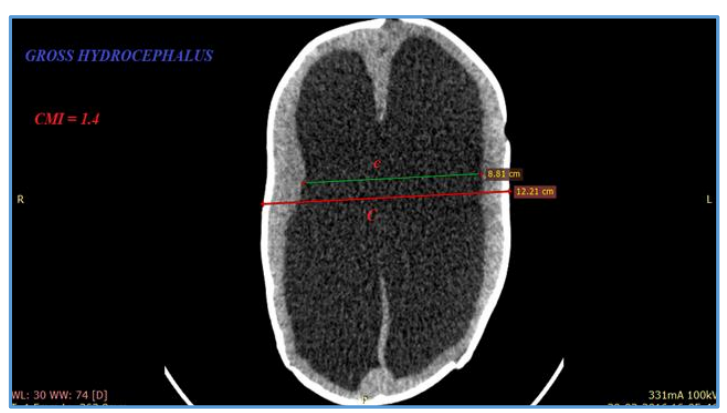

Figure 11. Illustrates Severe Hydrocephalus with CMI $<1.6$

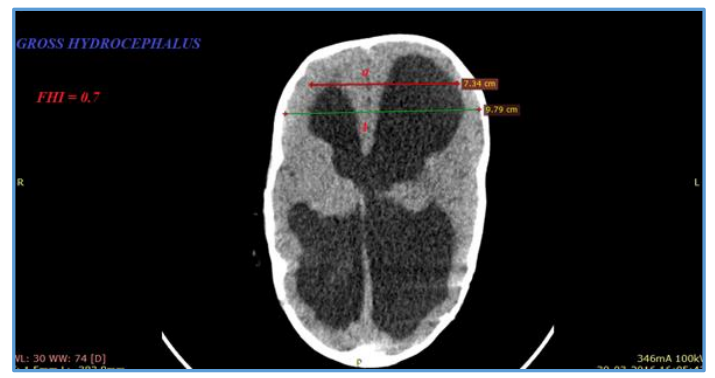

Figure 12. Illustrates Severe Hydrocephalus with 0.7 FHI

\section{DISCUSSION}

According to Y. Mondorf et al CMI is usually $>4.1$ in normal, 4.0 - 3.6 in mild cases, $3.5-3.0$ in moderate cases and $<2.9$ in severe hydrocephalus cases.[13] These measurements differed in our study, which graded mild hydrocephalus as $3.5-2.3$, moderate as $2.4-1.6$ and severe as $1.9-1.2$ on the basis of CMI. Based on our grading, CMI continued to reduce and FHI, EI, V/BP ratio up surged with increase in severity of hydrocephalus as shown in Table 2 .

\section{CONCLUSION}

Our study proved that combination of various indices measured together, rather than single index is better in grading hydrocephalus. CT is a valuable tool in differentiating communicating from non-communicating hydrocephalus depending on the level of obstruction. CT is also useful in determining the aetiology of hydrocephalus in children. Maximum of 19 patients had meningitis, 9 cases had HIE, 2 patients had infarcts and 1 each of DWS and TORCH. In 21 cases with non-communicating hydrocephalus, maximum of equal number of 7 patients had aqueductal stenosis and tumours respectively; 2 cases had HIE and 1 each of DWS, TORCH, granuloma, associated myelomeningocele and partial agenesis of corpus callosum respectively.

\begin{tabular}{|c|c|c|c|c|}
\hline Age & CMI & FHI & V/BP Ratio & EI \\
\hline < 1 Month & 3.05 & 0.462 & 0.35 & 0.373 \\
\hline 1 Month - 1 yrs. & 2.079 & 0.597 & 0.516 & 0.428 \\
\hline 1 - 5 yrs. & 3.087 & 0.418 & 0.313 & 0.427 \\
\hline 5 - 10 yrs. & 3 & 0.403 & 0.34 & 0.376 \\
\hline
\end{tabular}

Table 1. Different Ventricular Parameters versus Age Group

\begin{tabular}{|c|c|c|c|c|c|c|}
\hline Grade & Cases & Variant & V/BP & CMI & FHI & EI \\
\hline & & & & & & \\
\hline \multirow{2}{*}{ Normal } & 10 & Range & $<0.26$ & $5.5-2.3$ & $0.19-0.4$ & $0.2-0.45$ \\
\cline { 2 - 7 } & & Mean & 0.21 & 4.17 & 0.29 & 0.316 \\
\hline \multirow{2}{*}{ Mild } & 27 & Range & $0.26-0.4$ & $3.5-2.3$ & $0.3-0.6$ & $0.23-0.51$ \\
\cline { 2 - 7 } & & Mean & 0.34 & 2.7 & 0.453 & 0.383 \\
\hline \multirow{2}{*}{ Mod } & 11 & Range & $0.4-0.6$ & $2.4-1.6$ & $0.4-0.8$ & $0.24-0.7$ \\
\cline { 2 - 7 } & & Mean & 0.51 & 1.99 & 0.51 & 0.41 \\
\hline \multirow{2}{*}{ Severe } & 5 & Range & $0.6-0.9$ & $1.9-1.2$ & $0.7-1.1$ & $0.56-0.8$ \\
\cline { 2 - 7 } & & Mean & 0.76 & 1.42 & 0.88 & 0.73 \\
\hline
\end{tabular}

Table 2. Demonstrates Grading of Hydrocephalus based on all the Linear Indices

\section{REFERENCES}

[1] Hamidu AU, Olarinoye-Akorede SA, Ekott DS, et al. Computerised tomographic study of normal Evans index in adult Nigerians. Journal of Neurosciences in rural practice 2015;6(1):55-8.

[2] Patnaik P, Singh V, Singh S, et al. Lateral ventricle ratios correlated to diameters of cerebrum- a study on CT scans of head. J Anatomical Sciences 2014;22(2): 5-11.

[3] Wilk R, Kluczeunska E, Bajor G, et al. Normative values for selected linear indices of the intracranial fluid spaces based on CT images of head in children. Polish Journal of Radiology 2011;76(3):16-25.

[4] Choudhury AR. Infantile hydrocephalus: management using CT assessment. Childs Nerv Syst 1995;11(4):220-6.

[5] Pomschar A, Koerte I, Peraud A, et al. Hydrocephalus in childhood: causes and imaging patterns. Radiologe 2012;52(9):813-20.

[6] Kohlmeyer K, Shamena AR. CT assessment of CSF spaces in the demented and nondemented patients over 60 years of age. AJNR 1983;4(3):706-7.

[7] Venkatramana NK, Mukundan CR. Evaluation of functional outcomes in congenital hydrocephalus. Journal of Paediatric Neurosciences 2011;6(1):4-12. 
[8] Hahn FJ, Rim K. Frontal ventricular dimensions on normal computed tomography. AJR 1976;126(3): 492-6.

[9] LeMay M. Radiological changes of ageing brain and skull. AJNR 1984;5:269-75.

[10] Ragan DK, Cerqua J, Limbrick DD, et al. The accuracy of linear indices of ventricular volume in paediatric hydrocephalus: technical note. J Neurosurg Pedia 2015;15(6):547-51.

[11] Toma AK, Holl E, Kitchen ND, et al. Evan's index revisited: the need for an alternative in normal pressure hydrocephalus. Neurosurgery 2011;68 (4):939-44.
[12] Edwards J, Harvard Medical School, Gillian Liberman. Hydrocephalus in children: diagnostic imaging and radiological characteristics 2002.

[13] Mondorf Y, Gaab MR, Oertel J. The course of the cella media index according to schiersmann after ETV in different causes for obstructive hydrocephalus. German society of neurosurgery 2009. 